\title{
MULTI-PERSPECTIVE CHALLENGES ON COLLABORATIVE NETWORKS BUSINESS ENVIRONMENTS
}

\author{
Claudia-Melania Chituc \\ Americo Lopes Azevedo \\ Faculty of Engineering of the University of Porto (FEUP); INESC Porto
}

\{cmchituc;ala\}@fe.up.pt

\begin{abstract}
New forms of collaboration emerged as response to transformations of the business environment and the rapid information and communication technologies developments. In this context, collaborative networks rise as a powerful mechanism to achieve strategic objectives in a time response, quality and cost effective manner. The aim of this paper is to present the most relevant challenges concerning collaborative networks paradigm analysis, and to advance $a$ multi-perspective approach on collaborative networks (technological, semantic, social and business perspective), emphasizing the importance of the business view that allows collaborative networks to be regarded as combinations of inter-and intra-enterprise business processes. Balance Scorecard is seen as a powerful tool which can guarantee the strategic and business goal alignment within the network.
\end{abstract}

\section{INTRODUCTION}

The rapid development of information and communication technologies (ICT) and the transformations of the business environment determined new forms of collaboration, such as virtual enterprises, networked enterprises, or professional virtual communities. In this context, collaborative networks $(\mathrm{CN})$ emerge as a powerful mechanism to achieve competitiveness, world-excellence and agility in today's turbulent market conditions, comprising various heterogeneous entities with different competences, but symbiotic interests. This motivated numerous research projects and studies aiming at understanding and implementing this paradigm. Despite the myriad scientific results in the area of business networking and the socalled "collaborative work", the scientific community agrees that more work needs to be done (Camarinha-Matos, et al., 2004). Most studies on $\mathrm{CN}$ focus on technological aspects, such as supporting technologies, reference models, frameworks, infrastructures, often neglecting other issues, such as semantic, social and business aspects within $\mathrm{CN}$ (or partner organizations).

The aim of this paper is to address the most relevant challenges concerning $\mathrm{CN}$ paradigm analysis. This paper underlines the necessity to consider a multiperspective approach on $\mathrm{CN}$ (technological, semantic, social and business perspective), emphasizing the importance of the business view on $\mathrm{CN}$, that can be regarded as an aggregation of inter- and intra-enterprise business processes.

The paper is organized in five sections. After this introduction, the second section presents various types of networks arrangements usually considered by several researchers, and motives for participating in $\mathrm{CN}$. The third section proposes 
a multi-perspective approach on $\mathrm{CN}$ : technological, semantic, social and business perspective, with emphasis on the business view on $\mathrm{CN}$. The fourth section addresses issues on business processes in $\mathrm{CN}$ and business alignment. The fifth and last section contains the conclusions of the paper.

\section{COLLABORATIVE NETWORKS}

$\mathrm{CN}$ represent a valuable and effective approach to achieve strategic objectives in a time response and cost effective manner, with a high level of quality of delivery and customers' satisfaction, and generating value to stakeholders.

Current business trends determine a move from vertically integrated companies towards flexible network organizations, where the ability to quickly and efficiently set-up, maintain, develop and dissolve partnerships with business partners networkability - is a critical success factor (Osterle et. al., 2000). In fact, enterprises of all sizes are collaborating in order to fulfill client's demands, meeting their needs concerning cost, time response and quality, integrating various distributed business processes, and making relevant information available to all entities.

For the aim of this paper, in a broad sense, CN refers to a collection of heterogeneous organizations with different competences, but symbiotic interests, that join, efficiently combine, and coordinate their collective resources (i.e knowledge, skills, capital, assets, etc.) for a period of time in order to achieve a common objective, and use ICT to coordinate, develop and support their activities. The term $\mathrm{CN}$ is used in this article to represent collaborative forms such as virtual enterprises, extended enterprises, networked enterprises, professional virtual communities, and other emerging collaborative forms, with similar proprieties.

Miers (Miers, et. al., 1997) identified three main types of networks arrangements (Azevedo et al., 2002):

1. Dominant organization with a network of suppliers. This model is often used within large organizations, where one dominant player sets the main rules and uses a network of small firms to meet its needs and those of its customers. In this type of relationship, processes are usually focused on the repetitive supply of a service or product. The terms of the relationship are in general well defined in advance.

2. Co-operative network involves networks of small businesses undertaking together larger projects or services, which in turn may be supplied to larger or more dominant players. Processes are project-oriented and require a trust relationship between all co-operating entities.

3. Virtual teams. This type of network illustrates the trend of most large businesses to have teams managed on a project by project basis (seeking for a more agile structure).

Although an attractive option to undertake business, since they offer a number of advantages, $\mathrm{CN}$ face several difficulties, most obvious one being of technological nature, and referring mainly to issues such as inter- and intra-enterprise integration or interoperability. Alexakis (Alexakis et al., 2004) identified several inhibitors and challenges for the adaptation of virtual organization concept, such as: clear organization, adequate workforce, finance, communication, loss of competence, development of the partnership, low acceptance, and intellectual property.

Since the effort required to integrate heterogeneous entities is relatively high, several benefits must be attained to justify this effort. The literature on collaboration 
identifies some motives and benefits associated with inter-enterprise collaboration:

- to increase market share; to increase asset utilization; to enhance customer service - reduction in lead times, customer complains, etc.; to share and reduce the cost of product development; to reduce time in product development; to increase quality of product; to increase skill and knowledge; to enhance skill and knowledge; to have technological gain as participating firm; to achieve economies of scale in production (Lewis, 1990)

- to decrease risk of failure of product development (Parker, 2000)

- to reduce inventory - in the face of increasing technological complexity and rapid rate product development and obsolescence (Parker, 2000; Holton, 2001)

- to gain rapid access to markets (Parker, 2000; McCarthy et al., 2002)

- to increase flexibility; to attain international presence for small and medium size enterprises; to cope with changing dynamics; to have an effective knowledge management (Alexakis et al., 2004)

The authors identified also other motivations for inter-enterprise collaboration:

- to have access to resources (skills, knowledge, etc.) that are usually well beyond those of a single player

- to learn (gain knowledge) form other participants in the network

\section{MAIN PERSPECTIVES ON COLLABORATIVE NETWORKS BUSINESS ENVIRONMENT}

\subsection{Introduction}

CN comprise several heterogeneous and world-wide spread organizations, with different ICT solutions. Its power consists of this association of different enterprises and institutions with different aims and different competences, but symbiotic interests, in the form of a $\mathrm{CN}$. $\mathrm{CN}$ require tools, models and methodologies to support entities and employees to create just-in-time activity and exploit business opportunities.

The research undertaken allows the authors to consider four main perspectives on $\mathrm{CN}$, comprising several aspects of these rich and powerful business mechanisms:

1. technological perspective, referring to technologies for inter- and intraenterprise integration, interoperability, security, authentication, infrastructures supporting business, reference models and architectures, etc.;

2. semantic perspective, referring to semantic integration between business partners (data semantics, behavior semantics);

3. social perspective, which has a special emphasis on social actors (individuals and groups capable of performing activities and of interacting in order to pursue a specific goal);

4. business perspective concerning, among others, business alignment within the network, and inter- and intra-organization business processes.

Some other aspects of $\mathrm{CN}$ can be also considered, such as cognitive or knowledge perspective.

The following sub-sections detail the four above-mentioned main perspectives on $\mathrm{CN}$, with emphasis on the business perspective.

\subsection{Technological Perspective}

$\mathrm{CN}$ are usually analyzed from a technical perspective, with focus on aspects such as: 
messages format and content; activities for sending and receiving messages; specific negotiation messages and acknowledgement messages; time-out functionalities and catch-up attempts; supporting infrastructures, reference models and architectures.

In the context of $\mathrm{CN}$, technologies such as Peer-to-Peer (P2P), Web services, workflow, semantic Web, intelligent agents, grid are core solutions for enterprise integration and interoperability, streamlining transactions while supporting process coordination and consistency. Despite the relatively high number of infrastructures and ICT solutions claiming to support enterprise integration and the numerous scientific results in the business networking area, more work needs to be done since available solutions are usually cumbersome and lack in flexibility to respond to the most recent technological outcomes, focusing on very specific aspects, and do not guarantee a natural operational environment. They often separate and isolate particular departments, companies, supply chains, authorities, research institutions and each individual of their surroundings (Chituc, et al., 2005)..

Although intense research is being carried out on $\mathrm{CN}$ or related topics, and numerous distributed information technology infrastructures and distributed information systems architectures are available, the scientific community agrees that questions related to the formalization, conceptual development and semantic integration (namely concerning the formal description of the domain, ontology, behavior, etc.) are fundamental research topics waiting for a consistent development (Camarinha-Matos, 2003). The urgent need for new reference models, frameworks, business ICT solutions is illustrated also by several research projects, such as VOSTER (http://voster.vtt.fi) or THINKcreative (www.uninova.pt/thinkcreative ).

\subsection{Semantic Perspective}

Semantic perspective has little attention. Bussler (Bussler, 2003) launched as the grand challenge the issue of semantic integration among various organizations. Semantic integration encompasses data semantics and behavior semantics. Data semantics and ontologies are used to establish a formal semantic description of business domain concepts that allow an automatic transformation between them, without a human integration modeler. Process semantics concerns the match between communicating interfaces, so that their executions results in a consistent state after their execution is finished. In this context, when this grand challenge is met, the next (grander) challenge will be: how to achieve self-forming virtual enterprises, that is virtual enterprises where the detection of service providers, as well as their contracting is automated (Bussler, 2003).

\subsection{Social Perspective}

Social network analysis is not a completely new issue. The general theoretical framework of a networked society (Castells, 1996) determined intensive research developing and using networked-centric theories and methodologies. Wellman's work (Wellman et al., 1996) on cooperative work and tele-work is directly based on social network concepts. Gitell's research (Gitell, 2000) also highlights the role of social networks and the use of ICTs to support forms of relational coordination.

Despite the current research trends, according to (Soares et al., 2003), little attention has been paid to inter-organizational cooperation and the particular roles of the actors (individuals and groups). Nevertheless, relationships between enterprises will continue to involve people in key managing activities, taking operational, 
tactical and strategic decisions supported by conveniently distributed information systems.

\subsection{Business Perspective}

Business perspective in $\mathrm{CN}$ is often neglected. Research on business aspect of $\mathrm{CN}$ is scarce, and the few research studies available tackle very specific issues, such as value transactions in collaborative environments (Bititci, et. al., 2004) or accounting models of economic relationships in virtual enterprises (Belak, et.al., 2002).

$\mathrm{CN}$ analysis from a business perspective comprises business relationships within the network partners, as well as several attributes, such as:

- competences - refers to the expertise (skills, knowledge, etc.) one organization offers in a certain area/ domain, its capacity to disseminate and better advertise its capabilities, and also to its ways to make other entities discover its skills and knowledge;

- availability, which is concerned with its attainability in terms of negotiation, business operation, etc.

- cost - that concerns the shared production (development) cost, and also the costs to join the $\mathrm{CN}$

- value, which according to (Martinez, 2003) has two components: internal value (i.e. shareholder perspective, where value is synonym to wealth), and external value (i.e. customer perspective, where value refers to clients' satisfaction)

- functions and roles allocation and fulfillment. This refers to organizations' functions and roles allocated within the $\mathrm{CN}$, their performance, and functions and roles fulfillment during $\mathrm{CN}$ life-time and after their dissolution (i.e. support and maintenance services, exploitation strategies, etc.).

- IPR (Intellectual Property Rights) concerns, among others, issues related to the ownership of the information assets in a $\mathrm{CN}$

- trust that refers to the confidence among $\mathrm{CN}$ partners

- security (i.e. security of information shared or exchanged)

- business agreements that concerns a (formal or informal) contract among CN partners setting out the terms of the collaboration

- quality of service / quality of delivery refer mainly to Service Level Agreement (SLA) that represents a contract between a service provider and an end-user, which stipulates and commits the service provider to a required level of service.

- past performance is related to the reliability of the partners considering their past accomplishments

- legal issues concern aspects such as risk management, tax payment, etc.

- coordination and management. $\mathrm{CN}$ coordination assumes a critical role, since a $\mathrm{CN}$ comprises heterogeneous entities, with different goals and cultures, but symbiotic interests.

- development of new partnerships that could determine the creation of a new CN based on past fruitful collaborations

- business alignment. Business alignment concerns one enterprise's business activity performance, within the $\mathrm{CN}$, that should be in line with the activities developed by the rest of the partners.

The business perspective is of utmost importance within the $\mathrm{CN}$ and it is yet not fully explored, but has implication on technological, social and semantic aspects related to $\mathrm{CN}$. In the context of a business networking environment, a $\mathrm{CN}$ can be 
described as a network of business processes (as illustrated in Figure 1) that can be identified, documented, controlled and improved. For the aim of this paper, it was made a distinction between intra-business processes (considered private business processes - PvBP) and inter-business processes (considered public business processes - PuBP).

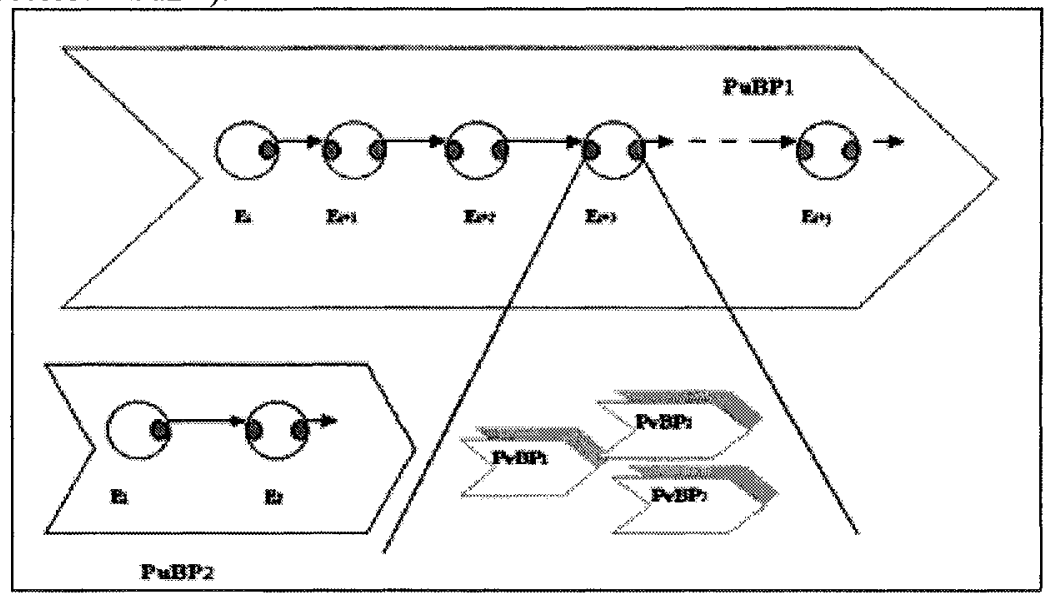

Figure 1. Collaborative Networks as Networks of Public and Private Business Processes

The main research questions inducing this paper are:

Question 1: Which business processes (inter- or intra- business processes) should one consider in order to operate the business model underlying the CN?

Question 2: How can one achieve an (technological and business) interface between several PuBP and a number of PvBP?

Question 3: How is it possible to align different PuBP with the strategic objectives of the $\mathrm{CN}$, and to obtain a win-win situation for all parties involved (partners and end-users)? Which methodology and tools should be used or developed?

Question 4: How can one evaluate performance in a business environment supported by a $\mathrm{CN}$ ?

\section{BUSINESS PROCESS ORIENTATION AND BUSINESS ALIGNMENT}

The process oriented organisation overcomes the problem found in traditional organizational structure where the information flows up the chain so that decisions can be made at the top. In fact, the goal of a process-oriented organization is to facilitate an "optimized" execution of processes.

However, such a process approach requires an effective management methodology and framework supported through a precision performance system to measure efficiency (resources consumed within the process) and effectiveness (quantify the ability of a process to deliver products or services according to their specifications).

The absence of meaningful goals and the use of wrong measures can drive organizations in the wrong direction. Thus, relevant perspectives and relevant 
objectives have to be selected and an adequate performance management system has to be designed and implemented. Nowadays, one of the trends is to consider a balanced approach to performance measure, namely taking into account past and future performance, financial and non-financial measures, soft and hard performance factors, internal and external indicators, etc. In this context, the Balanced Scorecard (BSC) concept plays an important and unquestionable role. The same applies to $\mathrm{CN}$ with probably a deeper interest due to the fact that in this environment interact different entities (autonomous, independent, heterogeneous, etc) which naturally limit the alignment level eventually necessary.

The BSC is an approach to performance measurement that combines traditional financial measures and instruments with non-financial measures to provide managers relevant information concerning the activities they are managing. First introduced in 1992 by Kaplan and Norton (Kaplan; Norton, 1992), the BSC concept has become widely known, and various forms of it have been widely adopted around the world. As Kaplan and Norton argue, no single measure can provide a clear performance target or focus attention on the critical areas of the business. The complexity of managing an organization today requires managers to view performance in several areas simultaneously. The selection of these performance measures should be linked to the organisation's strategic goals. One of the important characteristics of BSC design is the clustering of similar types of performance measures into groups (often called perspectives). In fact, BSC can be used, although requiring substantially different design and development processes, in two different applications:

1. Management control. BSC approach offers a holistic but focused view of performance measurement (extended to all business process);

2. Strategic control. BSC monitors the performance of all organisation as it implements activities associated with the implementation of strategic plan. The use of BSC in this context enables managers to establish their strategic objectives across a holistic view of the business, and to define relevant initiatives aligned with the goals defined at higher level.

In brief, BSC can be viewed as a multi-perspective strategic communication and performance measurement tool allowing translating strategy to key actions with the alignment of all relevant and critical activities. Concerning $\mathrm{CN}$, the BSC approach can be a powerful framework to guarantee the right strategic and business goal alignment within the network and at the same time can work as a 'performance contract' between all partners involved.

\section{CONCLUSIONS AND FURTHER WORK}

Although there are numerous studies and research projects on collaborative networks $(\mathrm{CN})$, most of them are focusing on technological aspects, undermining or even neglecting business, semantic or social aspects. No single perspective fully portrays or explains the complexity of a $\mathrm{CN}$, this is why a multi-perspective approach should be adopted when analyzing a collaborative business networking environment.

Besides underlining the importance of the technical, semantic and social views on $\mathrm{CN}$, this paper emphasizes the importance of the business perspective on a $\mathrm{CN}$ that can be regarded as a network of business processes. Thus, questions such as 
business process management, performance management and business process alignment are of most importance. In this context, the Balance Scorecard (BSC) becomes a powerful tool supporting a proper strategic and business goal alignment within a CN, while being a 'performance contract' among all partners.

Further work is being carried out in order to develop a holistic model on $\mathrm{CN}$, trying to identify major critical success factors.

Acknowledgements. The author C.M. Chituc would like to thank FCT (Fundação para a Ciência e a Tecnologia) for the $\mathrm{PhD}$ scholarship.

\section{REFERENCES}

1. Azevedo, A.L.; Toscano, C.; Sousa, J.P., "An Order Planning System to Support Networked Supply Chanins". In Collaborative Business Ecosystems and Virtual Enterprises, Kluwer, 2002

2. Alexakis, S.; Kolmel, B.; Heep, T., "VO in Industry: State of the Art". In Collaborative Networked Organizations: A Research Agenda for Emerging Business Models", Kluwer, 2004

3. Belak,V.; Kolakovic, M.; Kovacevic, B., "Economic Relationships between the Members of Virtual Enterprises". In Collaborative Business Ecosystems and Virtual Enterprises, Kluwer, 2002

4. Bititei, U.S.; Martinez, V.; Albores, P.; Parung, J., "Creating and Managing Value in Collaborative Networks". In International Journal of Physical Distribution \& Logistics Management, Emerald Group Publishing, Vol. 34 No. 3-4, 2004, p. 251-268

5. Bussler, C., "B2B Integration - Concepts and Architectures", Springer, 2003

6. Camarinha-Matos, L.M.; Afsarmanesh, H., "Collaborative Networked Organizations: A Research Agenda for Emerging Business Models", Kluwer, 2004

7. Camarinha-Matos, L., "New Collaborative Organizations and Their Research Needs". In Processes and Foundations for Virtual Organizations, Kluwer, 2003

8. Castells, M., "The Rise of the Networked Society", Vol. 1 of The Information Age: Economy, Society and Culture, Oxford: Blackwell, 1996

9. Chituc, C.M.; Azevedo, A.L., "Towards a Self-Forming Business Networking Environment", Proceedings ICEIS 2005 Conference (7th International Conference on Enterprise Information Systems), Miami (to be published in May 2005)

10. Gitell, J., "Organizing Work to Support Relational Coordination". In International Journal of Human Resource Management, 11(3), p. 517-34, Harvard University Press, 2000

11. Holton, J.A., "Building trust and collaboration in a virtual team". In Team Performance Management: An International Journal, Vol. 7 No. 3-4, 2001, p. 36-47

12. Kaplan, R.; Norton, D., "The Balanced Scorecard - Measures that Drive Performance", Harvard Business Review, January-February, p. 71-79, 1992

13. Lewis, D.J., "Partnership for Profit: Structuring and Managing Strategic Alliances", The Free Press, New York, NY, 1990

14. Martinez, V., "Understanding Value Creation: the Value Matrix and the Value Cube", PhD thesis, Strathclyde University, Glasgow, 2003

15. McCarthy, T.M., Golicic, S.L., "Implementing collaborative forecasting to improve supply chain performance". In International Journal of Physical Distribution and Logistic Management", Vol. 32 No. 6,2002, p. $431-54$

16. McLaren, T., Head, M.; Yuan, Y., "Supply chain collaboration alternatives: understanding the expected cost and benefits". In Internet Research: Electronic Networking Applications and Policy, Vol. 12 No. 4,2000 , p. $348-64$

17. Miers, D., Hutton, G. "Technology Futures for the World Wide Web - Business Needs Something Better", http://www cutter.com/concortium. Cutter Consortium

18. Osterle, H.; Fleisch, E.; Alt, R., "Business Networking: Shaping Enterprise Relationships on the Internet", Springer, 2000

19. Parker, A., "Interfirm collaboration and the new product development process", Industrial Management and Data Systems; Volume 100 No. 6; 2000

20. Soares, A.L.; Sousa, J.P.; Barbedo, F., "Modeling the Structure of Collaborative Networks: Some Contributions". In Processes and Foundations for Virtual Organizations, Kluwer, 2002, p. 23-30

21. Wellman, B.; Salaff, J.; Dimitrova, D.; Garton, L.; Haythornthwaite, C., "Computer Networks as Social Networks: Virtual Community, Computer Supported Cooperative Work and Telework", Annual Review of Sociology, No. 22, p. 213-38, 1996. 